\title{
Time Dependence of Transient Radiative Excess Carrier Lifetimes and Carriers Density in Indium Antimonide(InSb) Semiconductor in the Presence and Absence of Illumination
}

\author{
Getu Endale \\ Department of Physics, CNCS, Wolkite University, P.O.Box 007, Wolkite, Ethiopia
}

Copyright (C) 2019 by authors, all rights reserved. Authors agree that this article remains permanently open access under the terms of the Creative Commons Attribution License 4.0 International License

\begin{abstract}
In this work, we models the optical generation and transient radiative recombination excess carrier lifetimes in direct band gap semiconductors Indium antimonide (Insb) during illumination and after switching off the illumination. The time dependence of excess carrier density and excess carrier lifetimes are determined by using the doping level $10^{17} \mathrm{~cm}^{-3}$ and absorption rate $1.21 \times 10^{24} \mathrm{~cm}^{-3} \mathrm{~s}^{-1}$. The transient mean times for each excess carrier lifetimes to reach their steady-state values and excess carrier lifetime are determined.
\end{abstract}

Keywords Excess Carrier Lifetimes, Radiative Recombination, Direct Band Gap Semiconductor, Illumination

\section{Introduction}

Semiconductors are II-VI and III-V crystalline or amorphous solids with distinct electrical characteristics. Semiconductors in their natural state are poor conductors because a current requires the flow of electrons, and semiconductors have their valence bands filled, preventing the entry flow of new electrons. There are several developed techniques that allow semiconducting materials to behave like conducting materials, such as doping. An unbalanced number of electrons would cause a current to flow through the material [1].

A large direct band gap semiconductors are a semiconductors which their energy band gap ranged from $1 \mathrm{eV}$ to $3.5 \mathrm{eV}$ such as Gallium arsenide (GaAs). In direct band gap material such as Gallium arsenide and indium phosphide recombination is radiative considered as very important. Small direct band semiconductors with energy band gap ranged from 0.3 to $1.0 \mathrm{eV}$, like indium arsenide (InAs), gallium antimonide $\mathrm{GaSb}$, indium antimonide, InSb are characterized by the absence or small amount of impurity materials, presence of very high charge carrier concentration and high charge carrier mobility [2].
Generation in semiconductors refers to the process by which electron-hole pairs are created. Recombination refers to the inverse processes by which the electron-hole pairs are lost due to the spontaneous transition of an excited electron from conduction band to an unoccupied state in valence band. The excess energy and the change in the momentum released are either as photons or phonons or transferred to other carriers, which ensure energy and momentum conservation for the individual processes of electron hole recombination [3].

Evaluation of the excess (photo-generated) carriers' effective lifetime is very important in the determination of the photoconductivity of the semiconductors. Evaluation of the lifetime also requires knowledge of the determination of the carriers' mobility's, the thermal carrier concentrations for both holes and electrons and the determination of the density of photo-generated carriers which is directly related to the intensity and the spectral properties of the incident photon [4].

The concepts of carrier lifetime in semiconductor materials and average life expectancy in demography are similar. Like common mortals, electrons (and holes) die after some time and the average of this time is called the minority carrier life time. The free carriers' generation rate is affected by the photon flux density and the energy of illuminating sample; and the thickness of the sample. The absorbed photons create excess electron-hole pairs and as a result the densities of electrons and holes increase above their equilibrium value. This also results in the increment of the conductivity of material. This increment in the conductivity of the material due to illumination by light is called photoconductivity of material .The lifetime measurement is normally used in one way or another to assess material quality. High lifetimes are indicative of good material, while low lifetimes might point to problems such as inadequate crystal growth techniques that introduce dislocations or high concentrations of impurities in the semiconductor material [5].

The performance of semiconductor material depends on the lifetime of free charge carriers generated by the interaction of light with the material, referred to as the excess carriers' life- 
time. The excess carrier lifetime is in general determined by the recombination rate of the minority charge carriers in the bulk of the material and at the surface [6].

In this work, first the time dependence of the radiative recombination and generation excess carrier lifetimes in direct band gap Indium antimonide (Insb)semiconductors during illumination are determined, then the time dependence of the transient radiative recombination lifetime after and before switching off the illumination is determined.

The remaining parts of this work is organized as follows: Section two describes the theoretical formulation of transient radiative recombination lifetime after and before switching off the illumination. Section three deals with the numerical result and discussion of Time dependence of excess carrier density and Time dependence of radiative lifetime in Indium antimonide (Insb) semiconductors. Section four gives the summary and conclusion the work.

\section{Theory}

\subsection{Optical generation of carriers}

The photo-generated electrons and holes change the total electron and hole concentrations in the semiconductor to new values $n$ and $p$, respectively, given by:

$$
n=n_{0}+\delta n, p=p_{0}+\delta p
$$

where $n_{0}$ and $p_{0}$ are the densities of thermally generated electron and hole concentrations, respectively; and $\delta n$ and $\delta p$ are the photo-generated electron and hole concentrations, respectively.

The rate of optical generation of minority carriers is represented by $G_{0}$ and is given as the ratio of $\delta n$ to the radiative minority carriers' generation lifetime, $\tau_{G}$ :

$$
G_{0}=\frac{\delta n}{\tau_{G}}
$$

\subsection{Radiative recombination of carriers}

The total thermal recombination rate $R_{t h}$ is directly proportional to the product of the thermally generated concentration of electrons $n_{0}$ available in the conduction band and the thermally generated concentration of holes $p_{0}$ available in the valence band:

$$
R_{t h}=C_{R} n_{0} p_{0}
$$

Where $C_{R}$ is a constant known as the coefficient of holes in the valence band for the capture of electrons, and is related to the electron capture cross-section, $\sigma_{R}$ and the thermal velocity of the electron, $v_{t h}$ as

$$
C_{R}=\sigma_{R} v_{t h}
$$

The capture cross-section, $\sigma_{R}$ can be thought of as the volume swept out per unit time by a particle. When the dispersion in the dielectric constant is ignored, the band-to-band capture coefficient for bands with spherical symmetry was shown by van Roosbroeck and Shockley to depend on the absorption coefficient through the relation [7].

$$
C_{R}=e \pi \sqrt{k_{\infty}}\left(\frac{m_{n}}{m_{n}{ }^{*}+m_{p}{ }^{*}} \frac{300}{T}\right)^{\frac{3}{2}}\left(1+\frac{m_{n}}{m_{n}^{*}}+\frac{m_{n}}{m_{p}^{*}}\right)\left[E_{g}^{2}\right]
$$

where $e$ is the elemental charge and $k_{\infty}$, the high frequency dielectric constant of the material. $m_{n}$, rest electron mass, $m_{n}^{*}$, effective mass of electron, $m_{p}^{*}$, effective mass of hole, $T$, temperature of the system. The total non-thermal recombination rate $R$ during illumination is also directly proportional to the product of the non-thermal concentrations of electrons $n$ available in the conduction band holes $p$ available in the valence band:

$$
R=C_{R} n p
$$

where $n$ and $p$ are discussed in (1) above. The rate at which free photo-generated minority carriers disappear or recombine in this is represented by $U_{R}$ and is by the difference between the non-thermal recombination rate, $R$ and the thermal recombination rate, $R_{t h}$. From (3) and (6) in the absence of carriers $\operatorname{trap}(\delta p=\delta n)$ :

$$
U_{R}=C_{R}\left[\left(p_{0}+n_{0}\right) \delta n+\delta_{n}^{2}\right]=\frac{\delta n}{\tau_{R 0}}+C_{R} \delta_{n}^{2}
$$

Where $\tau_{R 0}$ is a time constant given by:

$$
\tau_{R 0}=\frac{1}{C_{R}\left(p_{0}+n_{0}\right)}
$$

For low injection level system, when $\delta n \ll p_{0}$, the quadratic term in $\delta n$ on the right side of (7) can be neglected and hence:

$$
U_{R}=\frac{\delta n}{\tau_{R 0}}
$$

For high injection level system, when $\delta n \gg p_{0}$, the linear term in $\delta n$ on the right side of (7) can be neglected and:

$$
U_{R}=C_{R} \delta_{n}^{2}
$$

\subsection{Radiative excess carrier lifetimes}

The radiative recombination rate of photo-generated minority carriers is also given as the ratio of the density of photogenerated electrons, $\delta n$ to the radiative minority carriers' recombination lifetime, $\tau_{R}[8]$.

$$
U_{R}=\frac{\delta n}{\tau_{R}}
$$

\subsubsection{Excess carrier lifetimes with illuminations}

The rate of accumulation of the photo-generated electrons in the conduction band is given by the difference between the free carriers' generation rate, $G_{0}$ and the recombination rates, $U_{R}$ :

$$
\frac{d \delta n}{d t}=G_{0}-U_{R}
$$

In general, the radiative excess carrier lifetime varies with time for high injection levels. In order to calculate the time dependence of $\tau_{R}$ and $\tau_{g}$, the relation describes the variation of 
$U_{R}$ with the excess carrier concentration, $\delta n,(6)$ is very important. Substitution of (7) into (12) gives [9]:

$$
\frac{d \delta n}{d t}=G_{0}-\left[\frac{\delta n}{\tau_{R 0}}+C_{R} \delta_{n}^{2}\right]
$$

Taking the initial free carrier density to be zero initially just when the illumination begins, (13) can be rearranged as:

$$
\int_{0}^{\delta n(t)} \frac{d \delta n}{\delta_{n}^{2}+\frac{\delta_{n}}{C_{R} \tau_{R 0}-\frac{G_{0}}{C_{R}}}}=-C_{R} \int_{0}^{t} d t
$$

Upon factorizing the denominator of the left side of (14) and integrating the term on the right side, we have:

$$
\int_{0}^{\delta n(t)} \frac{d \delta n}{\left(\delta_{n}+k_{1}\right)\left(\delta_{n}+k_{2}\right)}=-C_{R} t
$$

where $k_{1}$ and $k_{2}$ are arbitrary constants to be determined by comparing (14) and (15) as:

$$
k_{1}=\frac{2 G_{0} \tau_{R 0} \tau_{k}}{\tau_{R 0}-\tau_{k}}, K_{2}=-\frac{2 G_{0} \tau_{R 0} \tau_{K}}{\tau_{R 0}+\tau_{k}}
$$

Where $\tau_{k}$ is another time constant given by:

$$
\tau_{k}=\frac{\tau_{R 0}}{\sqrt{1+4 C_{R} G_{0} \tau_{R 0}^{2}}}
$$

Then by using partial fraction integration method (15) becomes:

$$
\int_{0}^{\delta n(t)}\left(\frac{A}{\delta_{n}+k_{1}}+\frac{B}{\delta_{n}+k_{2}}\right) d \delta n=-C_{R} t
$$

where $A$ and $B$ are also arbitrary constants. Upon comparing (15) and (18) and taking into accounts the constants in (16), we have:

$$
B=-A=C_{R} \tau_{k}
$$

Substitution of (19) into (18) and performing the integrations gives:

$$
\delta n(t)=\frac{2 G_{0} \tau_{R 0} \tau_{k}\left(1-\exp \left(-\frac{t}{\tau_{k}}\right)\right)}{\tau_{R 0}\left[1+\exp \left(-\frac{t}{\tau_{k}}\right)\right]+\tau_{k}\left[1-\exp \left(-\frac{t}{\tau_{k}}\right)\right]}
$$

The radiative recombination lifetime, $\tau_{R 1}$ with illumination is determined as function of time by using (20) along with (6) and (11) as:

$\tau_{R 1}(t)=\frac{2 \tau_{R 0} \tau_{k}\left[\tau_{R 0}\left(1+\exp \left(-\frac{t}{\tau_{k}}\right)\right)+\tau_{k}\left(1-\exp \left(-\frac{t}{\tau_{k}}\right)\right)\right]}{\left(\tau_{R 0}^{2}+\tau_{k}^{2}\right)\left[1-\exp \left(-\frac{t}{\tau_{k}}\right)\right]+2 \tau_{R 0} \tau_{k}\left[1+\exp \left(-\frac{t}{\tau_{k}}\right)\right]}$

The excess carrier generation lifetime is also determined using (20) and (2) as:

$$
\tau_{g}(t)=\frac{2 \tau_{R 0} \tau_{k}\left[1-\exp \left(-\frac{t}{\tau_{k}}\right)\right]}{\tau_{R 0}\left[1+\exp \left(-\frac{t}{\tau_{k}}\right)\right]+\tau_{k}\left[1-\exp \left(-\frac{t}{\tau_{k}}\right)\right]}
$$

Initially, for $t / \tau_{k} \ll 1$, by expanding the exponential terms in (20) to (22) and taking the first two dominant terms, we get:

$$
\begin{gathered}
U_{R}(t)=\frac{G_{0}}{\tau_{R 0}} t \\
\delta n(t)=G_{0} t \\
\tau_{R 1}(t)=\tau_{R 0}\left(1-\frac{\tau_{R 0}^{2}-\tau_{k}^{2}}{4 \tau_{R 0} \tau_{k}^{2}}\right) \\
\tau_{g}(t)=t
\end{gathered}
$$

For $t / \tau_{k} \rightarrow 0$,

$$
\begin{gathered}
U_{R}(t)=0 \\
\delta n(t)=0 \\
\tau_{g}(t)=0 \\
\tau_{R 1}(t)=\tau_{R 0}
\end{gathered}
$$

At steady-state, as $t \rightarrow \infty$, again, we have:

$$
\begin{gathered}
U_{R}(t)=G_{0} \\
\delta n=\frac{2 G_{0} \tau_{R 0} \tau_{k}}{\tau_{R 0}+\tau_{k}} \\
\tau_{R 1}(t)=\tau_{g} \approx \frac{2 \tau_{R 0} \tau_{k}}{\tau_{R 0}+\tau_{k}}
\end{gathered}
$$

\subsubsection{Excess carrier lifetime without illuminations}

On turning off the illumination (or $G_{0}=0$ ) after the system has reached a steady state after a total illumination time, $\tau,(11)$ becomes [10]:

$$
\frac{d \delta n}{d t}=-U_{R}=-\left(\frac{\delta n}{\tau_{R 0}}+C_{R} \delta n^{2}\right)
$$

Following the same procedure we used to integrate the differential equation in (13) to integrate the differential in (26) for $\delta n$ from the initial steady state value during illumination, (25) to $\delta n(t)$ and for the time from the total illumination time, $\tau$ to the later time, $t$, we get:

$$
\delta n(t)=\frac{4 G_{0} \tau_{R 0} \tau_{k}^{2} \exp \left(-\frac{t-\tau}{\tau_{R 0}}\right)}{\left(\tau_{R 0}+\tau_{k}\right)^{2}-\left(\tau_{R 0}^{2}-\tau_{k}^{2}\right) \exp \left(\frac{-(t-\tau)}{\tau_{R 0}}\right)}
$$

The recombination excess carrier lifetime in the absence of illumination in this case is also determined by substituting (27) into (11) as:

$$
\tau_{R 2}(t)=\tau_{R 0}\left[1-\frac{\left(\tau_{R 0}-\tau_{k}\right) \exp \left(-\frac{t-\tau}{\tau_{R 0}}\right.}{\tau_{R 0}+\tau_{k}}\right]
$$

Initially, for $(t-\tau) / \tau_{R 0} \ll 1$, we have:

$$
U_{R}(t)=G_{0}\left(1-\frac{t-\tau}{\tau_{R 0}}\right)
$$

$$
\begin{gathered}
\left.\delta n_{(} t\right)=\frac{2 G_{0} \tau_{R 0} \tau_{k}}{\tau_{R 0}+\tau_{k}}\left(1-\frac{\tau_{R 0}+\tau_{k}}{2 \tau_{R 0} \tau_{k}}(t-\tau)\right) \\
\tau_{R 2}(t)=\frac{2 \tau_{R 0} \tau_{k}+\left(\tau_{R 0}-\tau_{k}\right)(t-\tau)}{\tau_{R 0}+\tau_{k}}
\end{gathered}
$$

For $t-\tau \rightarrow 0$ : 


$$
\begin{gathered}
\delta n(t)=\frac{2 G_{0} \tau_{R 0} \tau_{k}}{\tau_{R 0}+\tau_{k}}, \\
\tau_{R 2}(t)=\frac{2 \tau_{R 0} \tau_{k}}{\tau_{R 0}+\tau_{k}}
\end{gathered}
$$

At steady state, when $t-\tau \rightarrow \inf$, we get from relations (27) and (28) that:

$$
\begin{gathered}
U_{R}(t)=0, \\
\delta n(t)=0, \\
\tau_{R 2}(t)=\tau_{R 0}
\end{gathered}
$$

As the changes of the quantities in (27) and (28) keep their initial values throughout the process after the switching of the illumination.

\section{Numerical Result and Discussion}

In this study, the time dependences of the radiative recombination and the optical generation excess carrier lifetimes during illumination and after switching off the illumination in $\mathrm{InSb}$ sample are determined. The transient properties of all the excess carrier lifetimes before attaining the steady-state conditions are described in detail.

\subsection{Time dependence of excess carrier density}

Figure 1 depicts the time dependence (a) optical carrier generation rate and; excess carrier concentration in InSb sample of doping level $10^{17} \mathrm{~cm}^{-3}$ when it is illuminated by photons of optical carrier generation rates (b) $10^{18} \mathrm{~cm}^{-3} \mathrm{~s}^{-1}$ (c) $8 \times 10^{14} \mathrm{~cm}^{-3} \mathrm{~s}^{-1}$ and (d) $10^{19} \mathrm{~cm}^{-3} \mathrm{~s}^{-1}$. The time dependent of the normalized $G_{0}$ in Figure 1 (a) is drawn by keeping its magnitude constant at 1 for the total time interval between 0 and $\tau$ to represent constant illumination and; then by making the magnitude of $G_{0}$ zero to represent the switching off the illumination in the next time interval between $\tau$ and $2 \tau$.

The time dependent of the excess carrier densities in Figure 1 (b) to (d) are also drawn using the expression of the time dependence excess carrier density in (20) during illumination for the time interval between 0 and $\tau$; and the expression of the time dependence excess carrier density in (27) after switching of the illumination for the time interval between $\tau$ and $2 \tau$. To normalize the excess carrier densities the obtained results are divided by the respective maximum constant values of the excess carrier densities at steady state during illumination described in (25).

When the semiconductor is under constant illumination of relatively low injection level as in Figure 1 (a) for time, $\tau$, the absorbed photons also generate EHPs at constant rate. Hence, the concentration of EHPs must linearly increase with time in the initial stage (for time $t$ close to 0 ) after switching on the light as illustrated by line $L_{1}$ in Figure 1 (b). That is, for $t$ close to 0 , the excess carrier concentration is very small and the effect of recombination is negligible; a constant $G_{0}$ dominates the accumulation process. Recombination, on the other hand, tries to reduce the excess carrier concentrations to zero.
As more EHPs are generated, the recombination rate starts to influence the accumulation process and the rate at which excess carriers accumulate (as in (12)) decreases exponentially and finally becomes zero (at steady-state). As the value of the recombination rate increases and approaches $G_{0}$ at steady state, the low injection level excess carrier density during illumination reaches its maximum constant value at steady-state as shown in Figure 1 (b).
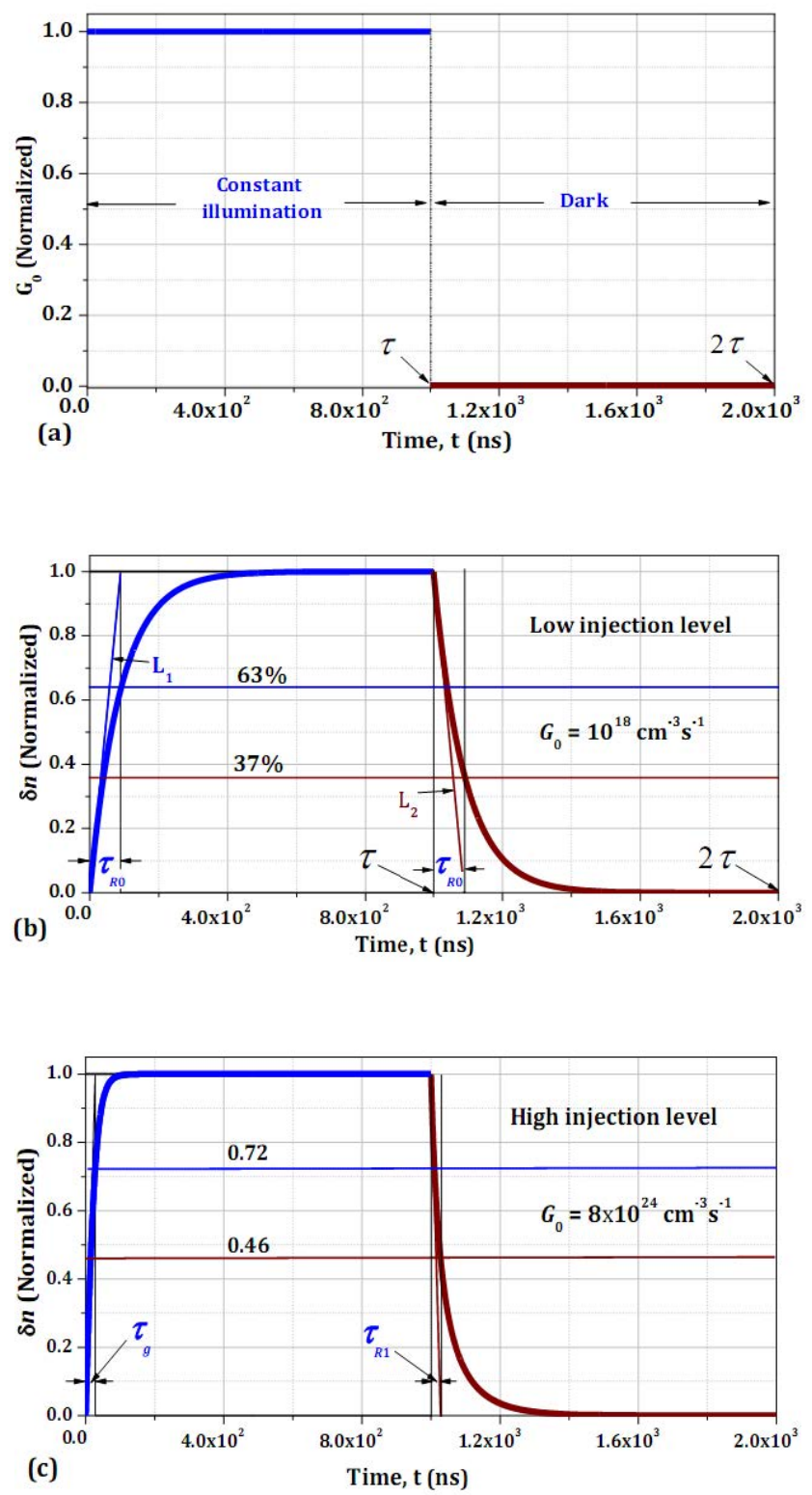

The transient mean time, $\tau_{R 0}$ for $\delta n(t)$ to attain its maximum value in a system under low injection level during illumination is hence, defined as the time in which $\delta n(t)$ increases from 0 to 63 percent of its value at the steady-state, or the time taken for $\delta n(t)$ to rise to its steady-state value, if the carriers continue to increase at the initial rate of accumulation. 


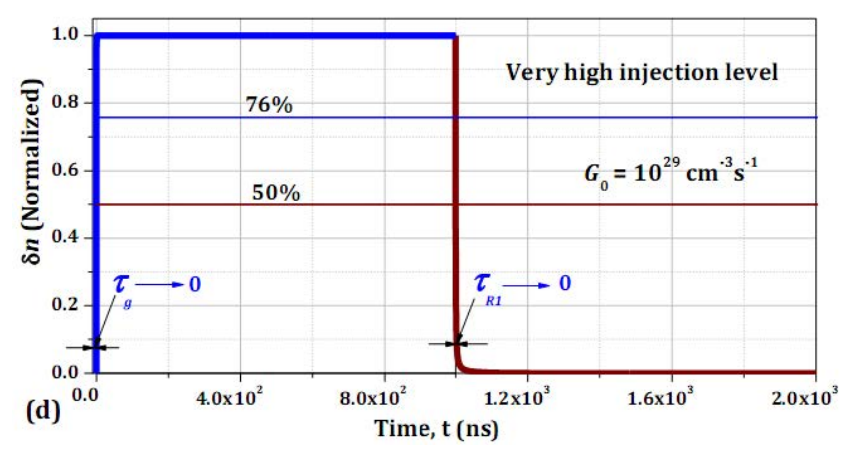

Figure 1. Variations of the normalize (a) optical carrier generation rate and; excess carrier concentration in $I n S b$ sample of doping level $10^{17} \mathrm{~cm}^{-3}$ when it is illuminated by photons of optical carrier generation rates (b) $10^{18} \mathrm{~cm}^{-3} \mathrm{~s}^{-1}$ (c) $8 \times 10^{14} \mathrm{~cm}^{-3} \mathrm{~s}^{-1}$ and (d) $10^{19} \mathrm{~cm}^{-3} \mathrm{~s}^{-1}$.

Upon turning the illumination off $\left(G_{0}=0\right)$ after the system has reached a steady-state after time, $\tau$, only the recombination rate dominates the entire process. The decay rate is very fast at the beginning and the time dependence of the excess carrier density can be approximated as linear under low injection level. If the recombination rate keeps its initial value throughout the process, $\delta n(t)$ will fall to 0 in a time equal to the low injection level steady-state value excess carrier lifetime, $\tau_{R 0}$. However, since the excess carrier concentration decreases, subsequent slower decay follows and $\delta n(t)$ drops exponentially to 37 percent of its maximum initial value in time, $t-\tau=\tau_{R 0}$. The transient mean time, $\tau_{R 0}$ for $\delta n(t)$ in a dark system in this case is therefore defined as the time taken for the excess carrier concentration to decrease to 37 percent of its maximum value, or the time taken for excess carrier concentration to decrease to zero, provided the carriers continue to decay at their initial rate of recombination. As the injection level increases, the steadystate value of $\delta n(t)$ for a system under illumination increases.

The transient mean times for $\delta n(t)$ to reach the steadystate during illumination and after switching of the illumination remain the same and their value are reduced to that of the steady-state values of $\tau_{g}$ and $\tau_{R 1}$ described in (25) depending on the level of injection. As the injection (illumination) level highly increases, the transient mean times for $\delta n(t)$ to reach the steady-state during illumination and after switching of the illumination goes to zero as depicted in Figure 1 (c) and (d). The ranges of both the steady-state conditions during illumination and after switching of the illumination for $\delta n(t)$ increase with increasing the injection level as shown in Figure 1 (c) and (d). For a system under slightly high injection level during illumination as the one shown in Figure $1(\mathrm{c}) . n(t)$ increases from 0 to $72 p e r c e n t$ of its value at the steady-state, in the mean transient time, $\tau_{g}$ given in (25), while it drops exponentially to 46percent of its maximum initial value in the same time after switching of the illumination. For a system under very high injection level during illumination, $\delta n(t)$ increases from 0 to 76 percent of its value at the steady-state, in a very small transient mean time, $\tau_{g}$ is shown on the left side of Figure 1 (d). For a system undergoing very high injection level, $\delta n(t)$ also drops exponentially to 50percent of its maximum initial value in a very small transient mean time after switching of the illumination as illustrated on the right side of Figure 1 (d).

\subsection{Time dependence of excess carrier lifetimes}

Figure 2 depicts the time dependences of (a) radiative recombination carrier lifetime during illumination, $\tau_{R 1}$ (blue line), (b) optical generation carrier lifetime, $\tau_{g}$ (green line) and (c) radiative recombination carrier lifetime after switching off the illumination, $\tau_{R 2}$ (wine line). The transient mean times for each excess carrier lifetimes to reach their steady-state values are shown at the initial stages of each graph. The doping level of the sample used is $10^{17} \mathrm{~cm}^{-3}$ and the absorption rate used is $1.21 \times 10^{24} \mathrm{~cm}^{-3} \mathrm{~s}^{-1}$.
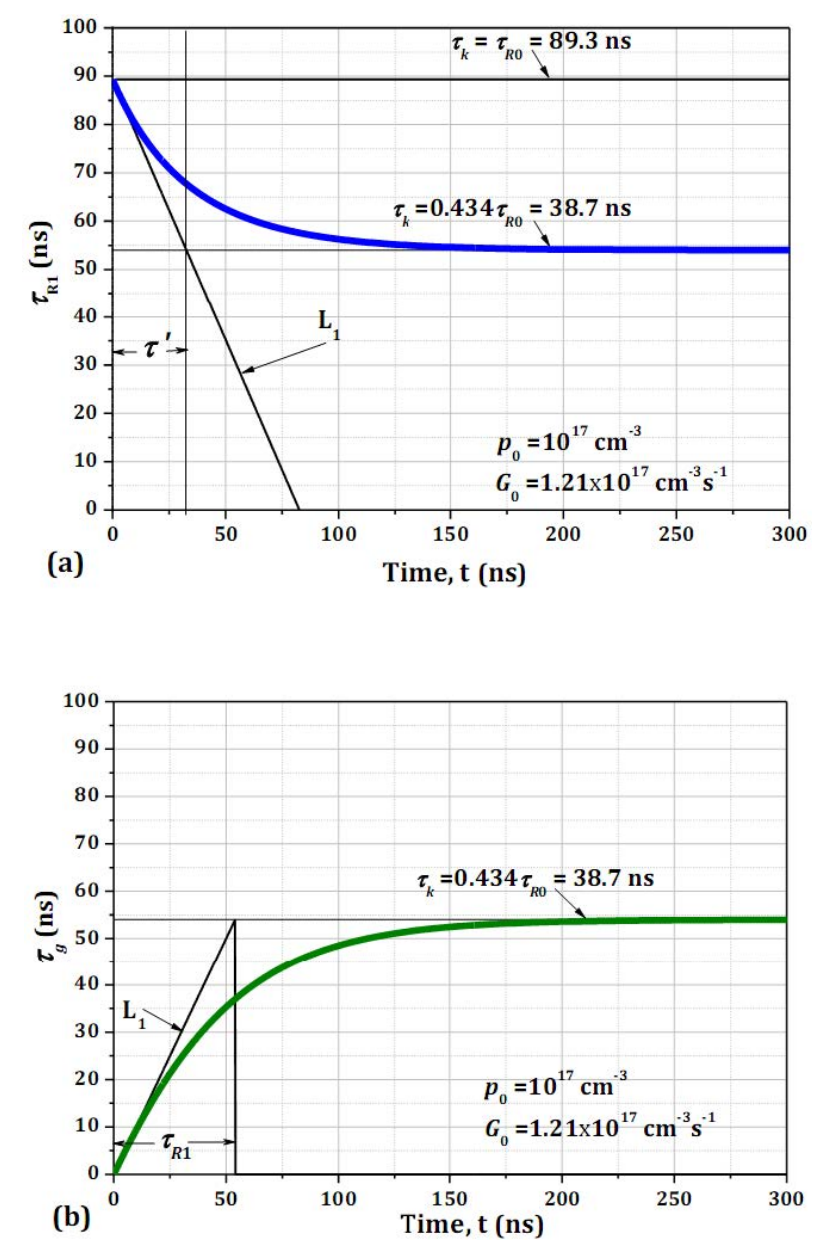

In general, photo-generated carriers can be recombined both with opposite photo-generated carriers and thermallygenerated carriers in the system. The net excess carrier recombination rate in (7) above is hence the sum of the recombination rate of a free carrier with opposite photo-generated and thermally-generated carriers. The net recombination rate of photo-generated carriers with other opposite photo-generated carriers is dominant in high injection level system as depicted in (10), while the net recombination rate of photo-generated carriers with the thermally generated carriers is dominant in low injection level system as shown in (9). As a result, all the excess carrier lifetimes $\tau_{R 1}(t), \tau_{g}(t)$ and $\tau_{R 2}(t)$ remain a function of $G_{0}$ in high injection level system and only a function of $\tau_{R 0}$ in low injection level system. The maximum excess carrier density obtained using (25) by the illumination of photon absorption rate $1.21 \times 10^{24} \mathrm{~cm}^{-3} \mathrm{~s}^{-1}$ is approximately 


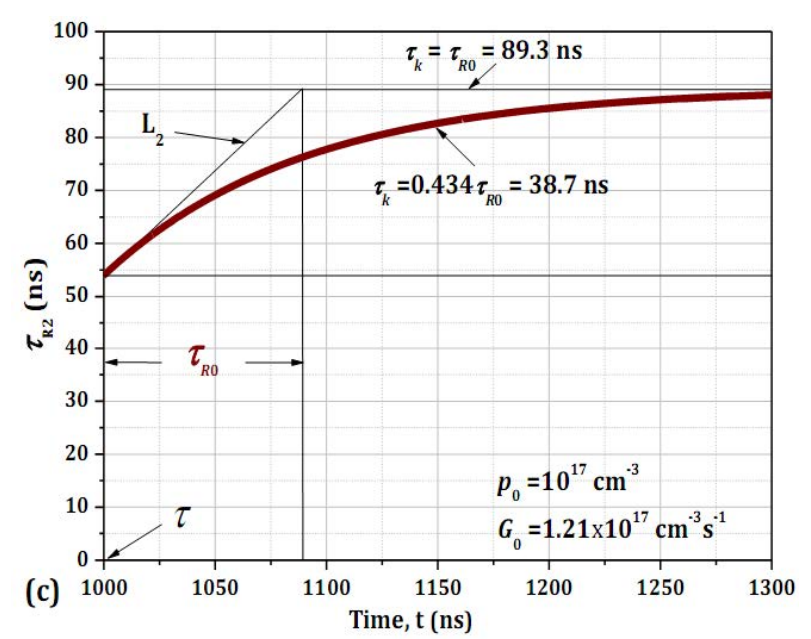

Figure 2. Time variations of (a) radiative recombination carrier lifetime during illumination, $\tau_{R 1}$ (blue line), (b) optical generation carrier lifetime, $\tau_{g}$ (green line) and (c) radiative recombination carrier lifetime after switching off the illumination, $\tau_{R 2}$ (wine line). The transient mean times for each excess carrier lifetimes to reach their steady-state values are shown at the initial stages of each graph. The doping level of the sample used is $10^{17} \mathrm{~cm}^{-3}$ and the absorption rate used is $1.21 \times 10^{24} \mathrm{~cm}^{-3} \mathrm{~s}^{-1}$

$6.53 \times 10^{16} \mathrm{~cm}^{-3}$ and the value of the time constant, $\tau_{k}$ described in (17) has value $38.7 \mathrm{~ns}$ for all the curves in Figure 2 (a) to (c). Hence, the photo-generated carrier density is comparable with thermally generated carrier density and the system can be considered as slightly under high injection level. Initially, when the time, $t$ approaches zero, the system can be considered as under low injection level.

The recombination excess carrier lifetime during illumination, $\tau_{R 1}$ depicted in Figure 2 (a) hence decreases with time from its maximum value $\tau_{R 0}$ (which is equivalent to the low injection value) to the steady state value described in relation (25). The generation excess carrier lifetime, $\tau_{g}$ illustrated in Figure 2 (b) also increases with time from its maximum value zero to the steady state value in (25). Both $\tau_{R 1}$ and $\tau_{g}$ have the same steady state values. However, the recombination excess carrier lifetime after switching of the illumination, $\tau_{R 2}$ shown in Figure 2 (c), increases with time from its high injection level value described in relation (25) at time, $\tau$ to the low injection value, $\tau_{R 0}$ at steady state. If the variation of $\tau_{R 1}(t)$ with time keeps its initial condition as in (23) throughout the process, it reaches its value at steady-state described in (25) in the time interval as indicated by $L_{1}$ in Figure 2 (a). Similarly, if the variation of $\tau_{g}(t)$ with time keep its initial condition as in (23) throughout the process, it reaches its steady-state value described in (25) in the time interval described in (25) as indicated by lines $L_{1}$ in Figure 2 (b). If the variation of $\tau_{R 2}(t)$ with time keeps its initial condition throughout the process, it reaches its value at steady-state, $\tau_{R 0}$ in the time interval, $\tau_{R 0}$ as indicated by $L_{2}$ in Figure 2 (c).

Figure 3 illustrates the effects of injection level on the time dependences of (a) radiative recombination carrier lifetime during illumination, $\tau_{R 1}(t)$ (blue line), (b) optical generation carrier lifetime, $\tau_{g}(t)$ (green line), (c) radiative recombination carrier lifetime after illumination is switched off, $\tau_{R 2}(t)$ (wine line) and (d) when the excess carrier lifetimes ${ }_{R 2}(t), \tau_{g}(t)$ and
$\tau_{R 2}(t)$ are plotted all together for the sample used in Figure 2 above. In this case, three injection levels of optical carriers generation rates $10^{18} \mathrm{~cm}^{-3} \mathrm{~s}^{-1}, 1.21 \times 10^{24} \mathrm{~cm}^{-3} \mathrm{~s}^{-1}$ and $2.2 \times 10^{25} \mathrm{~cm}^{-3} \mathrm{~s}^{1}$ that are considered as low, slightly high and very high injection levels, respectively. The values of the resulting time constant, $\tau_{k}$ corresponding to each illumination levels are $89.3 \mathrm{~ns}, 38.7 \mathrm{~ns}$ and $10 \mathrm{~ns}$, respectively as shown in Figure 3 (a) to (c).
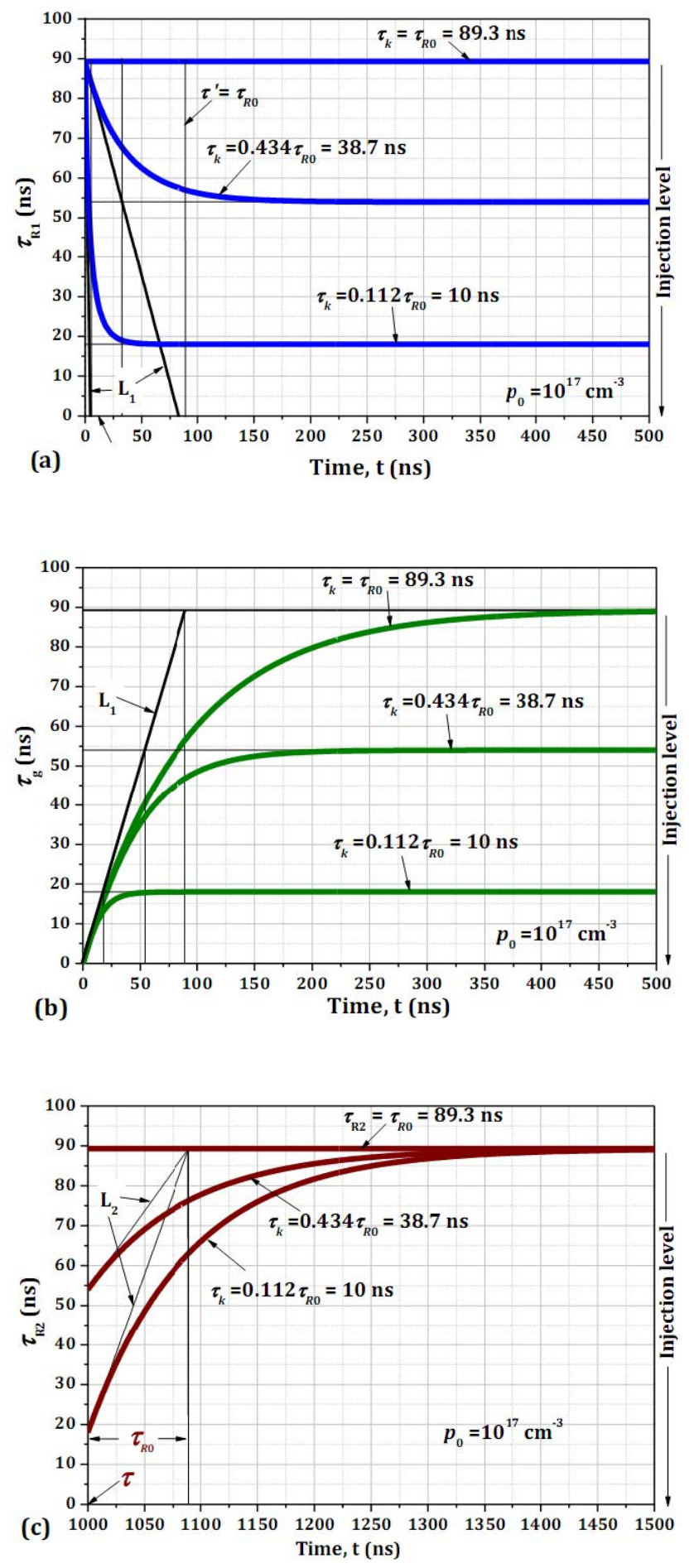


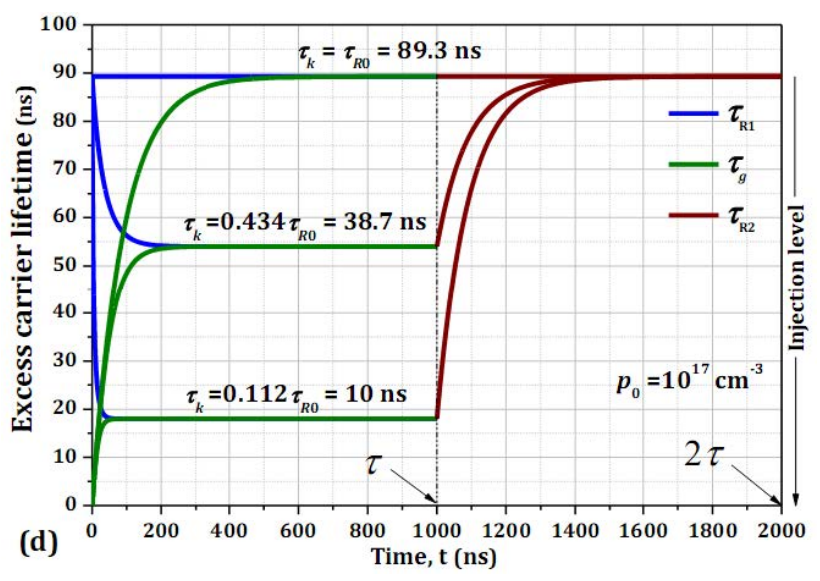

Figure 3. Effects of injection level on the time dependences (a) for radiative recombination carrier lifetime during illumination, $\tau_{R 1}$ (blue line), (b) for generation carrier lifetime, $\tau_{g}$ (green line), (c) for radiative recombination carrier lifetime after illumination is switched off, $\tau_{R 2}$ (wine line) and (d) when the excess carrier lifetimes $\tau_{R 1}, \tau_{g}$ and $\tau_{R 2}$ are plotted all together for the sample used in Figure 2 above. The sample is illuminated by photons of three injection levels of optical carrier generation rates of $10^{18} \mathrm{~cm}^{-3} \mathrm{~s}^{-1}, 1.21 \times 10^{24} \mathrm{~cm}^{-3} \mathrm{~s}^{-1}$ and $2.2 \times 10^{25} \mathrm{~cm}^{-3} \mathrm{~s}^{-1}$ that are considered as low, slightly high and very high injection levels, respectively.

As it can be seen the figure, the variations of $\tau_{R 1}(t)$ and $\tau_{R 2}(t)$ with time at the initial time for different injection levels are also varying, while the variation of $\tau_{g}(t)$ with time at the initial time remains the same for all injection levels. This is illustrated in Figure 3 (a) and (c) with slanted lines represented by $L_{1}$ and $L_{2}$ of different slopes tangent to the curves of $\tau_{R 1}(t)$ and $\tau_{R 2}(t)$ at the beginning for different injection levels. However, all the curves of $\tau_{g}(t)$ in Figure 3 (b) for different injection levels are parallel to each other at the initial time and the slanted line $L_{1}$ with slope 1 shown on the left side of the figure represents the similarity of the variation of all the curves with time at the beginning. The slope of line, $L_{1}$ at the beginning of Figure 3 (a) is varying with injection levels and given by $-\left(\tau_{R 0}^{2}-\tau_{k}^{2}\right) / 4 \tau_{k}^{2}$. The slope of $L_{1}$ is zero for low injection system $\left(\tau_{R 0}=\tau_{k}\right)$ and increases with increasing the injection level since $\tau_{k}$ decreases with injection level. The slope of line, $L_{2}$ at the beginning of Figure 3 (c) is also varying with injection levels and given by $\left(\tau_{R 0}-\tau_{k}\right) /\left(\tau_{R 0}+\tau_{k}\right)$. The slope of $L_{2}$ also zero at low injection level and increases with increasing the injection level until it reaches the value of 1 for very high injection level (see (41)). This leads to the proof of the premise that low injection level radiative recombination excess carrier lifetime is constant and has value $\tau_{R 0}$ which is controlled only the thermal equilibrium properties of the material; and the low injection level optical generation excess carrier lifetime increases with time.

Figure 3 (d) shows the relation between the radiative recombination lifetime and the optical generation lifetime. As can be seen from the figure, the low injection level recombination excess carrier lifetime remains constant at time, $\tau_{R 0}$ for the entire time interval between 0 and $2 \tau$, where $\tau$, is the total illumination time. However, the low injection level optical generation excess carrier lifetime exponentially increases from zero to its steady-state value $\tau_{R 0}$. As the injection level increases, the recombination excess carrier lifetime exponentially decreases and reaches its steady state value described in (25); and as the illumination is switched off at time $\tau$, the recombination excess carrier lifetime exponentially increases and reaches its steady state constant value, $\tau_{R 0}$. After time $2 \tau$, the full process is repeated. The high injection level optical generation excess carrier lifetime again exponentially increases from zero to its steady-state value described in (25) until time $\tau$. Optical generation excess carrier lifetime does not exist between times $\tau$ and $2 \tau$, because of there is no illumination. When each The constant value at very low injection level and the increasing of the values of the recombination excess carrier lifetime at the beginning $(t=0)$ and after switching off the illumination for the system under high injection level as shown in Figure 3 (d) indicates that, a semiconductor performs more when it is under low injection level.

\section{Summary and Conclusion}

In this work the time dependences of the radiative recombination and the optical generation excess carrier lifetimes during illumination and after switching off the illumination in InSb are determined. The transient properties of all the excess carrier lifetimes before attaining the steady-state conditions are described in detail. The optical excess carrier generation rate $G_{0}$ is kept at constant non-zero value during illumination and kept at zero after switching of the illumination. Electron-hole pairs (EHPs) are continuously generated under illumination, and the excess charge carrier concentrations in the respective bands are increase. As more EHPs are generated, the recombination rate starts to influence the accumulation process and the rate at which excess carriers accumulate is exponentially decreases. As the value of the recombination rate increases and approaches $G_{0}$ at steady state, the generation lifetime, $\tau_{g}(t)$ also approaches to $\tau_{R}$. The photo- generated carriers are recombined both opposite photo-generated carriers and thermally - generated carriers in the system. The net recombination rate of photo-generated carriers with other opposite photogenerated carriers is dominant in high injection level. For a system undergoing very high injection level, $\delta n(t)$ also drops exponentially to 50percent of its maximum initial value in a very small transient mean time after switching of the illumination.

The photo-generated carrier density is comparable with thermally generated carrier density and the system can be considered as slightly under high injection level. The ranges of both the steady-state conditions during illumination and after switching of the illumination for $\delta n(t)$ increase with increasing the injection level. The optical generation excess carrier lifetime does not exist between times $\tau$ and $2 \tau$, because of there is no illumination. In low injection level the radiative recombination excess carrier life time is constant and has value $\tau_{R 0}$, which is controlled only the thermal equilibrium properties of the material. After switching off the illumination the recombination excess carrier lifetime exponationally increases and reaches its steady state constant value, $\tau_{R 0}$. 


\section{Acknowledgements}

We would like to thanks our family and friends for their moral and psychological support during the preparation of this manuscript.

\section{REFERENCES}

[1] Mehta,V.K, Principles of Electronics, ed., 2008-01-01, p. 56, (6 December 2015).

[2] B.K., " Large-Band-gap Semiconductors," Tr. J. of Physics;, no. 2, (1999).

[3] A. Cuevas, D. Macdonald, Measuring and interpreting lifetime of silicon wafers, Solar Energy 76 255262, (2004).

[4] H. Gebrehiwet, "Absorption Coefficient and Dielectric Function of Direct Band Gap Silicon Nanocrystallities,", pp,(200-204), (years,2006).

[5] Tr,Large band gap Semiconductors, vol. 23, pp. 577-582, (1999).

[6] C. Measurement, "Measurement of the Band Gap in Silicon and Germanium," in applied physics, pp. (48-199), (Years, 1980).

[7] G.S .Nolas, J. Sharp and J. Gold Smid, Thermo electrics: Basic principles and new materials developments (Springer science and busines midia) (2013).

[8] D.K.schroder ,semiconductor material and Device characterization, 3rd (IEEE press; wiely,[Piscataway, NJ];Hobo ken,N.J,2006)

[9] J.H. Bahk and A. Shakouri, Appl. Phys. Lett. 105, 52-106 (2014).

[10] Goswami, A. Thin film fundamentals. New Age International pvt Itd , (2008). 